\title{
Fragile and Brittle Bone Disease or Osteogenesis Imperfecta: A Case Report
}

\author{
Navin H Krishnamurthy ${ }^{1}$, Nagarathna Chikkanarasaiah ${ }^{2}$, Aishwarya Nanjappa ${ }^{3}$, Nimishabalakrishnan Vathariparambath ${ }^{4}$
}

\begin{abstract}
Aim and objective: The aim and objective of this report is to describe the dental management of 11-year-old patient with type III osteogenesis imperfecta (OI).

Background: Osteogenesis imperfecta or brittle bone disease is caused by mutations in the collegen type I gene which is a heterogeneous rare connective tissue disorder. Dentinogenesis imperfecta, hearing impairment, scoliosis, sclera is blue, hyperlaxity of ligaments, and fragile skin are other common features. Individuals having positive family history suggest a straightforward diagnosis of Ol but can be difficult in the absence of affected family.

Case description: We report a case of 11-year-old boy, with a chief complaint of pain and swelling on the lower left back tooth region which was associated with extraoral draining sinus. His medical history revealed multiple fractures sustained during routine handling. On examination, the child was pale, dyspneic, with rhizomelic dwarfism, and relative macrocephaly with frontal bossing. On the grounds of history taken, clinical examination and respective investigations carried out, we came to a conclusion of osteogenesis imperfect type III. As the child needs a special care, we planned for a conservative treatment approach.

Conclusion: The craniofacial abnormalities in OI-III affected person's impact on their dentofacial appearance and masticatory function. A detailed dental and craniofacial investigation is necessary in affected persons in order to identify any primary or secondary abnormalities. As soon as the deciduous teeth erupt, patients with Ol should be evaluated with adequate dental treatment and oral hygiene instructions in order to reduce the need for extensive treatment.

Clinical significance: Although oral manifestations are seldom seen, dentist should be extremely alert while managing this fragile bone disease. Early diagnosis, increased awareness, and effective treatment plan will reduce the effects of this debilitating disease. An ounce of prevention is worth a pound of cure, especially when something has no cure.
\end{abstract}

Keywords: Connective tissue disease, Inherited disease, Osteogenesis imperfecta type III.

International Journal of Clinical Pediatric Dentistry (2020): 10.5005/jp-journals-10005-1792

\section{BACKGROUND}

Osteogenesis imperfecta $(\mathrm{Ol})$ is a rare inherited disorder affecting connective tissue integrity, which is characterized by bone fragility of varying severity ranging from lethal forms to those with very minimal features. ${ }^{1}$ The incidence is between 10,000 and 20,000 live births. The craniofacial abnormalities in affected persons can also impact on their dentofacial appearance and masticatory function. Care should be taken while managing such patients, as there is increase chance of bone fracture, and early diagnosis and effective treatment plan will reduce the effects of this debilitating disease.

\section{Case Description}

Here, we are presenting a rare case of a 11-year-old boy referred to our Department of Pediatric and Preventive Dentistry with the chief complaint of pain and swelling on the lower left back tooth region from the past 20 days (Fig. 1). Swelling was initially small in size gradually progressed to present size and associated with extraoral draining sinus for which he consulted a private dental clinic. Although he was under medication for past 1 week, there was no reduction in the size of the swelling (Fig. 2). His medical history revealed multiple fractures sustained during routine handling with difficulty in walking. His birth and neonatal period were uneventful. Family history revealed a positive finding of the same illness in his elder sibling. On examination, child was sick looking, pale, dyspneic, with rhizomelic dwarfism, and relative macrocephaly with frontal bossing. There was bilateral bowing of lower limbs with varus
${ }^{1-4}$ Department of Pedodontics and Preventive Dentistry, RajaRajeswari Dental College and Hospital, Bengaluru, Karnataka, India

Corresponding Author: Navin H Krishnamurthy, Department of Pedodontics and Preventive Dentistry, RajaRajeswari Dental College and Hospital, Bengaluru, Karnataka, India, Phone: +91 9845272314, e-mail: navina30@yahoo.co.in

How to cite this article: Krishnamurthy $\mathrm{NH}$, Chikkanarasaiah $\mathrm{N}$, Nanjappa A, et al. Fragile and Brittle Bone Disease or Osteogenesis Imperfecta: A Case Report. Int J Clin Pediatr Dent 2020;13(4):425-428.

Source of support: Nil

Conflict of interest: None

deformity and flexion deformity of the thumb. Patient profile was concave with typical triangular faces with fragile skin (Fig. 1). He had white sclera with narrow thoracic cage. Abdomen was protuberant with marked kyphosis. Patient had undergone surgery for impaired vision and hearing was normal. There was marked scoliosis with convexity toward left. Asymmetry was seen on the left side of the face with palpable submandibular lymph nodes, which was mobile and tender on palpation. On inspection, a solitary diffuse swelling was present on the left side of the face measuring $2 \times 3$ $\mathrm{cm}$, extending from the corner of the mouth to middle third of the mandible anteroposteriorly, and from ala-tragal line to the base of the mandible superior-inferiorly, associated with draining sinus. The swelling was soft in consistency, fluctuant, mild-tender on 
palpation, and there was a localized rise in temperature. On intraoral examination, swelling present in relation to 75 extending from attached gingiva up to buccal vestibule (Fig. 3). Deep dentinal caries was present in relation to mandibular left primary second molar which was tender on percussion and presented with vestibular tenderness and obliteration. The intraoral lesion was rubbery and fluctuant on palpation. Tooth present was 155312112122258442 413132 75. Dental caries with respect to 11,21, 15 and 25, caries with pulp exposure with respect to 75,84 . Intraoral periapical radiograph and orthopantomograph and cone-beam computed tomography were taken (Fig.4), and finally, diagnosis has arrived as dentoalveolar abscess with respect to 75 associated with extraoral draining sinus and pathological fracture of body of mandible on the left side. Investigation with hemogram revealed microcytic hypochromic anemia with relative lymphocytosis, echocardiography showed mild pulmonic regurgitation renal function test, and electrolytes were normal. Reduced Serum calcium level. Alkaline phosphatase was significantly raised, bilateral pneumonitic patches and haziness

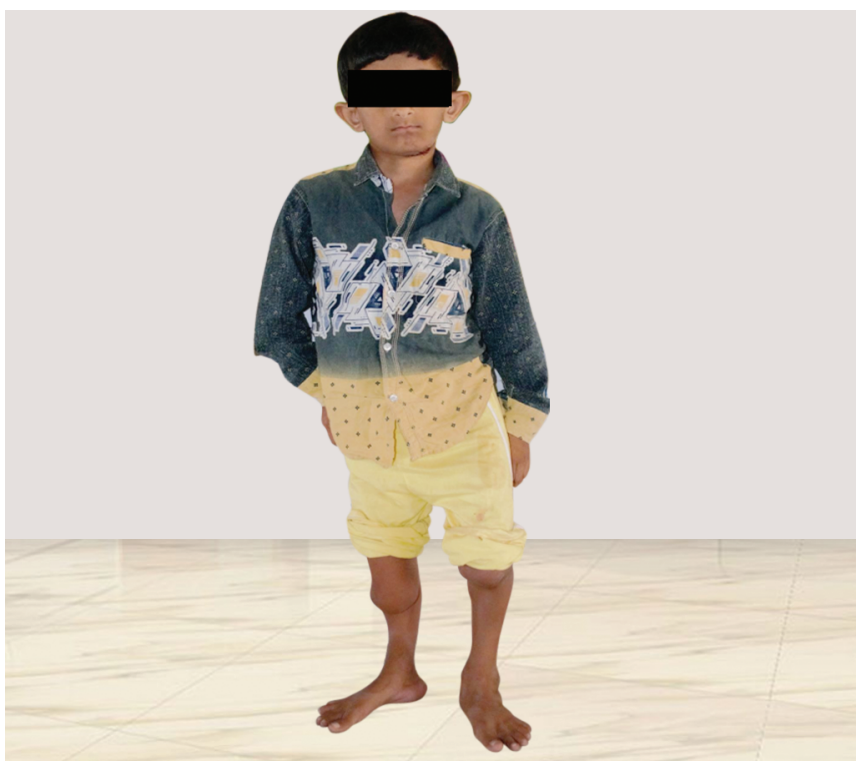

Fig. 1: Patient photograph showing typical features of osteogenesis imperfect

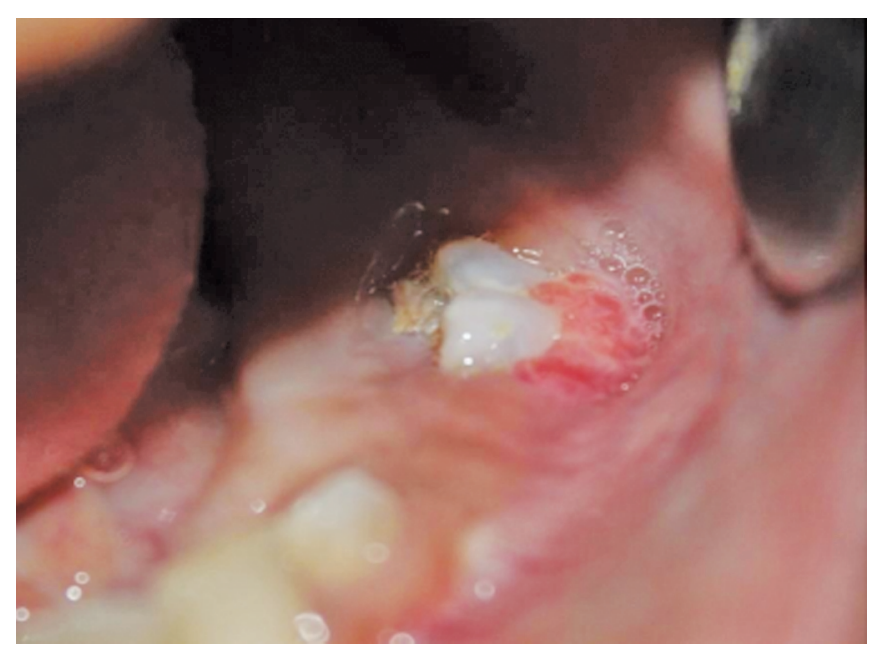

Fig. 3: Intraoral photograph showing submerged 75 with deep dental caries and obliteration in the vestibule with lack of air spaces seen in chest X-ray. Hip X-ray revealed protrusion acetabulum. X-ray of the extremities showed thinned cortices (Figs 5 and 6). X-ray spine shows platyspondyly. Owing to his physician's high index of suspicion, a diagnosis of Ol-III was pursued, which is the non-lethal form of the disease.

Calcium and vitamin D therapy was initiated, and an in-depth discussion regarding bisphosphonates was pursued. Advised oral prophylaxis and oral hygiene instructions, lesion sterilization and tissue repair were done in relation to 75 , pulp therapy with respect to 84 , and restoration in relation to $15,11,21$, and 25 were done. Tablet Augmentin 375 mg, Soframycin ointment, and tablet Metrogyl-200 mg were prescribed for 5 days. Patient was recalled after 3 days, 10 days, 15 days, 1 month, 3 months, and 4 months. Clinically healing extraoral sinus was noticed, and intraorally the findings were satisfactory and erupting 43 was noted (Fig. 7).

\section{Discussion}

On the grounds of history taken, clinical examination and respective investigations carried out, we came to a conclusion of OI-III. Our patient presented with short stature, significant bowing, recurrent fractures, typical triangular facies, hypermobile

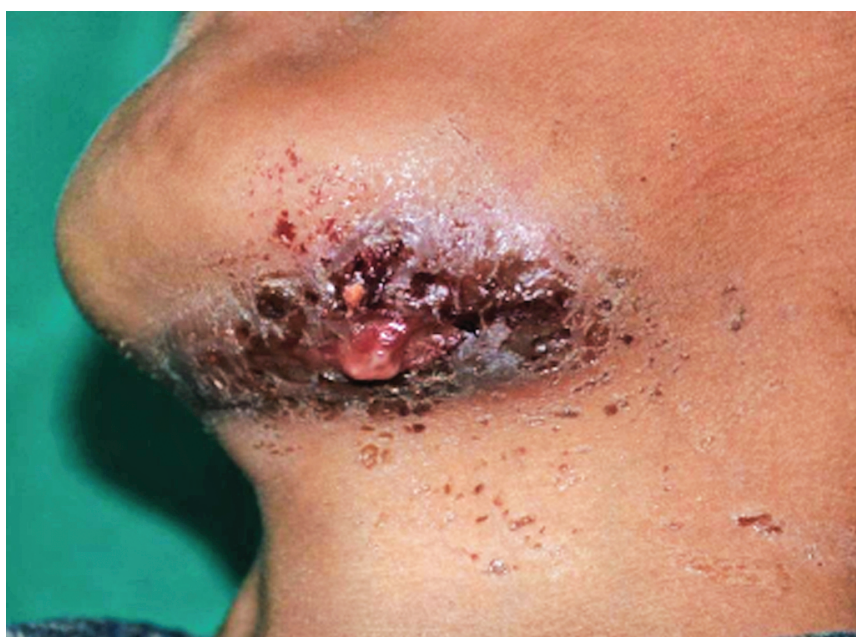

Fig. 2: Preoperative photograph showing extraoral draining sinus

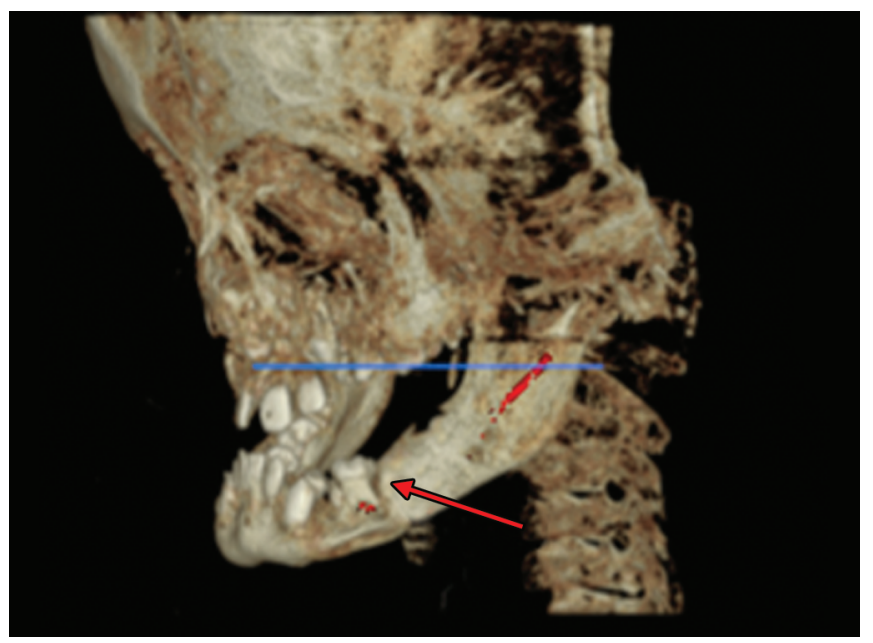

Fig. 4: Cone-beam computed tomography image showing lingually tilted 75 associated bone resorption and pathological fracture of body of mandible and thinning of lower border of mandible 

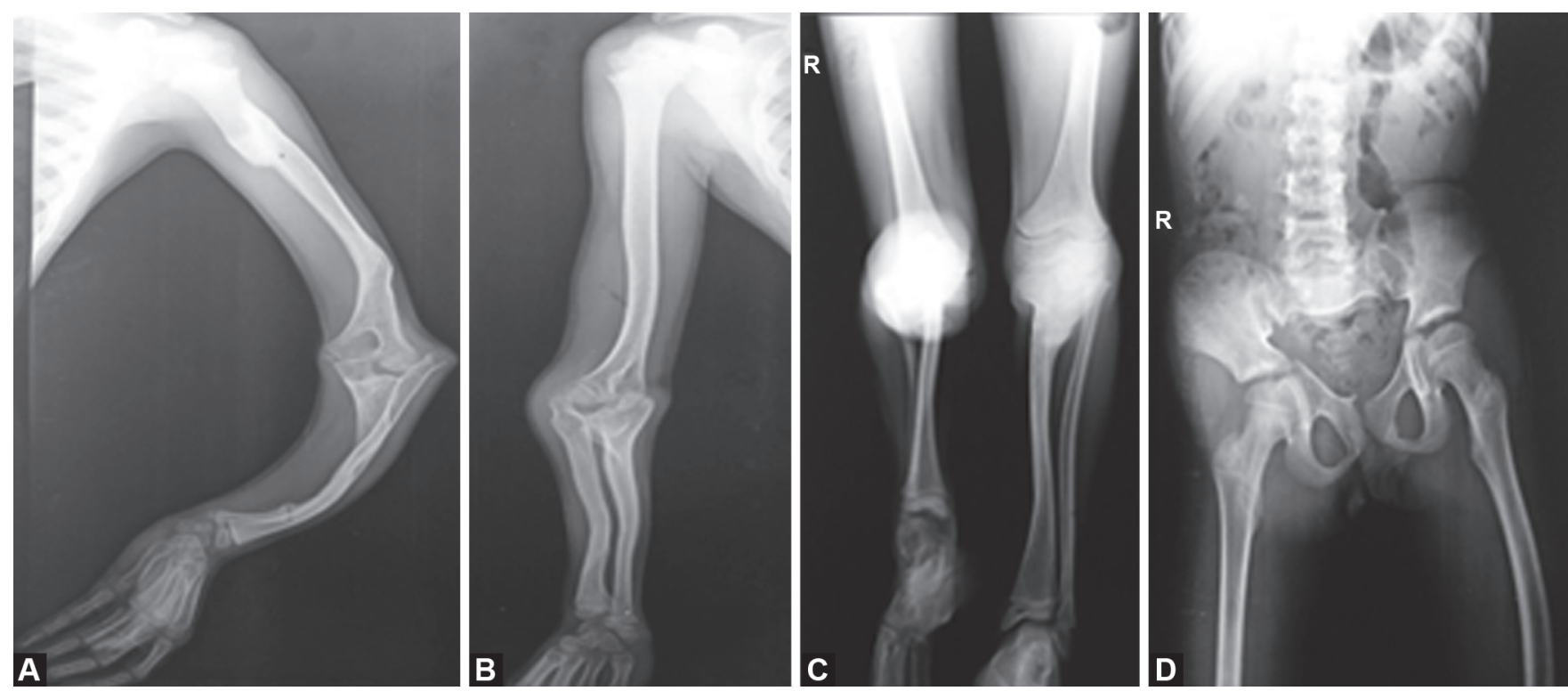

Figs $5 A$ to $D$ : X-ray of long bones showing multiple healed and healing fractures with reduced trabecular pattern with thinning of the cortex
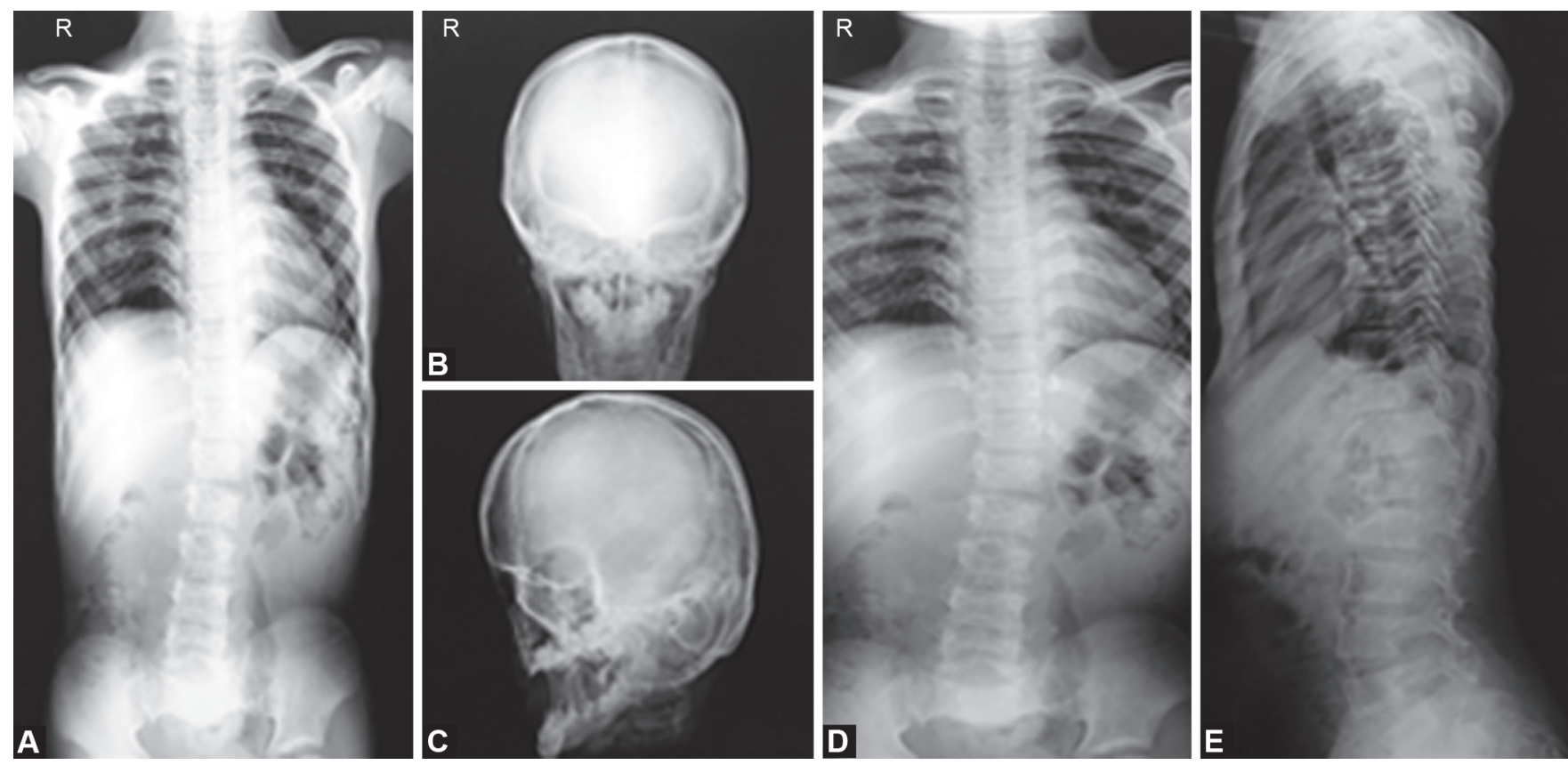

Figs 6 A to E: X-ray showing AP spine, platyspondyly and scoliosis and chest showing multiple pneumonitic patches. Hip X-ray revealed protrusion acetabulum

joints, skeletal deformities, cardiac abnormalities, hearing loss, hematopoietic changes, respiratory difficulty due to rib cage deformities, and fragile skin except for blue sclera which all are the classic features ${ }^{2}$ of Ol-type III. This condition may or may not be associated with dentinogenesis imperfecta leads to enamel breakage due to abnormal collagen. The lower teeth are more severely affected than the upper teeth. ${ }^{1}$ According to Sillence classification, ${ }^{3}$ this is constituted by four types (I to IV) based on clinical and radiological features without the possibility of curative clinical treatment. ${ }^{4}$ Novel types $\mathrm{V}, \mathrm{VI}$, and VII in which type I collagen genes were not involved were later added. The sillence classification is universally accepted to classify the degree of OI. ${ }^{5}$
Reasonable therapeutic possibilities are capable to improve the course of the disease and quality of life. ${ }^{6,7}$

\section{CONCLUSION}

- In order to identify primary and secondary abnormalities in individual, detailed dental and craniofacial investigation is necessary.

- Multispecialty approach is necessary in the management of individuals with OI type III.

- Adequate dental treatment and oral hygiene instructions are necessary in order to reduce the need for extensive treatment. 


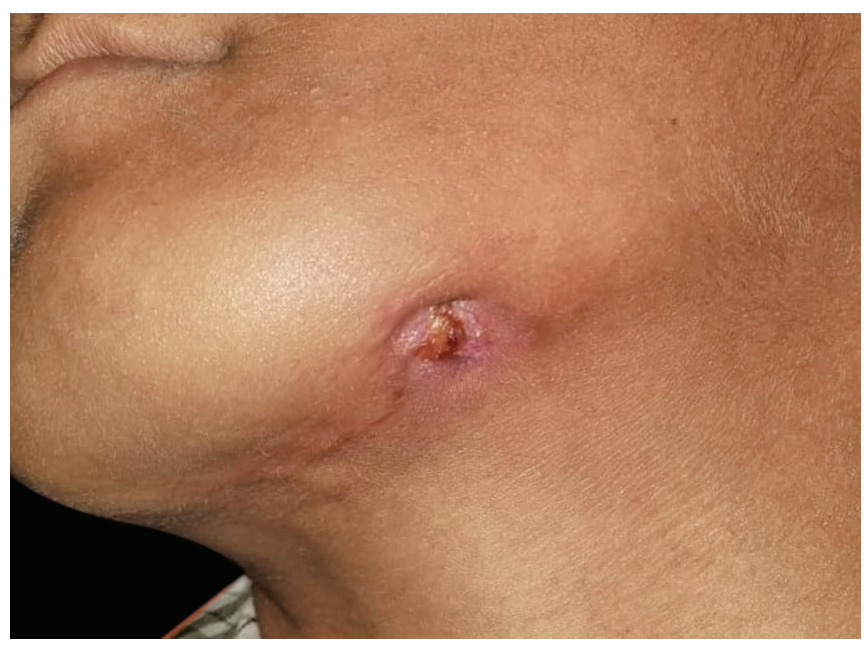

Fig. 7: Followup photograph after 4 months

\section{Clinical Significance}

Although oral manifestations are seldom seen, dentist should be extremely alert while managing this fragile bone disease and sometimes pedodontist are the first identifier of such rare diseases. Early diagnosis, increased awareness, and effective treatment plan will reduce the effects of this debilitating disease. "An ounce of prevention is worth a pound of cure...especially when something has no cure".

\section{Acknowledgments}

We would like to extend our sincere thanks to staffs and postgraduate students, Department of Pediatrics, RajaRajeswari Medical College and Hospital, Bengaluru, for their most valuable contribution. We also extent our gratitude to the management of RajaRajeswari Dental College and Hospital, Bengaluru, Karnataka, India, for their constant encouragement and support.

\section{References}

1. Kamalammal R, Venkatamurthy M, Bandari AK. Osteogenesis imperfecta type 111. Int J Contempor Pediat 2016;3(1):268-270.

2. Beattie ML, Kim JW, Gong SG, et al. Phenotypic variation in dentinogenesis imperfecta/dentin dysplasia linked to 4q21. J Dent Res 2006;85(4):329-333. DOI: 10.1177/154405910608500409.

3. Pillion JP, Vernick D, Shapiro J. Hearing loss in osteogenesis imperfecta: characteristics and treatment considerations. Genetics Research International 2011;2011:1-6. DOI: 10.4061/2011/983942.

4. Bastos F, Perez LT, Narváes CP, et al. Severe osteogenesis imperfecta: case report. Einstein (São Paulo) 2010;8(4):480-482. DOI: 10.1590/ s1679-45082010rc1863.

5. van DijkFS, Cobben JM, Kariminejad A, et al. Osteogenesis imperfecta: a review with clinical examples. Mol Syndromol 2011;2(1):1-20. DOI: 10.1159/000332228.

6. Fano V, Rodríguez Celin M, Del Pino M, et al. Osteogenesis imperfecta. Clinical, functional and multidisciplinary evaluation of 65 patients. An Pediatr (Barc) 2010;72(5):324-330.

7. Van Dijk FS, Pals G, Van Rijn RR, et al. Classification of osteogenesis imperfecta revisited. Eur J Med Genet 2010;53(1):1-5. DOI: 10.1016/ j.ejmg.2009.10.007. 